\title{
APPLICATION OF TARGET DETECTION ALGORITHM BASED ON DEEP LEARNING IN FARMLAND PEST RECOGNITION
}

\author{
Shi Wenxiu and Li Nianqiang \\ School of Information Science and Engineering, University of Jinan, Jinan, China
}

\begin{abstract}
Combining with deep learning technology, this paper proposes a method of farmland pest recognition based on target detection algorithm, which realizes the automatic recognition of farmland pest and improves the recognition accuracy. First of all, a labeled farm pest database is established; then uses Faster R-CNN algorithm, the model uses the improved Inception network for testing; finally, the proposed target detection model is trained and tested on the farm pest database, with the average precision up to $90.54 \%$.
\end{abstract}

\section{KEYWORDS}

Object detection algorithm, Faster R-CNN, Inception network.

\section{INTRODUCTION}

For a large agricultural country, the production status of rice, wheat, corn, soybean and other crops plays an important role in the stable development of the national economy. In recent years, the government has paid more and more attention to the development of science and technology in agriculture and has continued to increase investment in this area. However, agricultural production is still easily affected by the environment, Climate change, environmental pollution, agricultural disasters and improper management and other factors will cause crop yield reduction, especially the impact of pests in farmland is more serious. In this case alone, the annual agricultural economic losses in Europe reach 28.2\%, North America reaches 31.2\%, and the economic losses in Asia and Africa are as high as $50 \%^{[1]}$. Traditional pest identification and diagnosis methods mainly rely on manual identification ${ }^{[2]}$, which is closely related to the comprehensive quality of professionals. The subjective factors have a great influence on the accuracy and timeliness of pest control, which is not conducive to the effective work of farmland pest control. Therefore, it is necessary to study a fast and low-cost automatic detection method of farmland pests.

Li Wenbin ${ }^{[3]}$ used a single pixel background segmentation method based on RGB color to segment rice pest images, and used SVM support vector machine to identify and classify. Compared with traditional recognition methods, the recognition rate is better, but the number of pest image samples is small, and the identification type is single. Pan Chunhua ${ }^{[4]}$ and others used a grid search algorithm to improve the search efficiency of the image target pest area, and used SVM to train multiple classifiers to identify four major vegetable pests, with an average recognition rate of $93 \%$. Yang Guoguo ${ }^{[5]}$ used Otsu algorithm to find threshold adaptively to complete image segmentation. The SVM classifier was used to classify and identify the Chinese 
rice locust, with an accuracy rate of $88.3 \%$.Yang Wenhan ${ }^{[6]}$ used Canny operator and Otsu threshold segmentation method to segment 15 cotton pests, and used binary tree classification and support vector machine for classification and recognition, and the recognition effect was good.

The actual environment of real-life farmland pest classification is very complicated, and there are many types of pests. In order to achieve more effective and wider application of farmland pest detection technology, this paper combines deep learning and field pest detection, and proposes a field pest identification method based on target detection algorithm, which greatly improves the accuracy of field pest detection.

\section{MODEL BUILDING}

\subsection{Farmland Pest Database}

A good sample set is the basis of image recognition research ${ }^{[7]}$. Because no public data for farmland pest detection is currently available, this article has collected 2,472 farmland pest image samples through the Internet for the goals and tasks of pest recognition. In order to prevent overfitting due to insufficient data during training, This paper uses the following two data expansion methods for training samples: (1)In the stage of image preprocessing, some image space transform operations are used, the original image is randomly rotated, translated, horizontally flipped, scaled, and sheared. (2)This paper adds salt and pepper noise to the training image. This method randomly obtains the pixels of the image and sets them as high brightness points and low gray points. The schematic diagram of sample set expansion is shown in Figure 1.

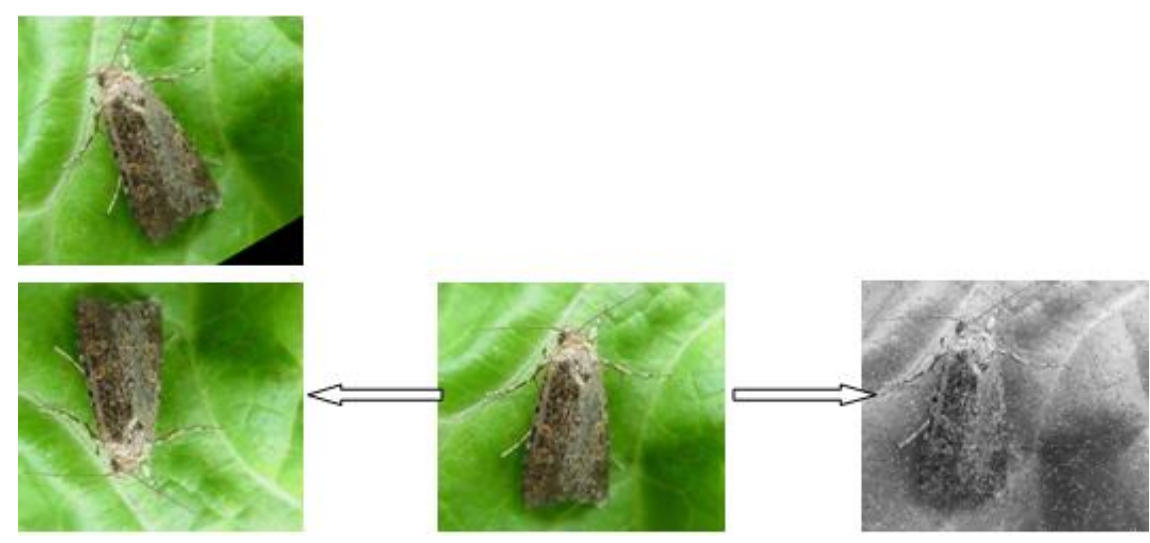

Figure 1. Image rotation (left) and Noise (right)

Finally, the farmland pest data set containing 10 categories was sorted out, The expanded sample capacity reached 12474 , as listed in Table 1 
Table 1. Heading and text fonts

\begin{tabular}{|l|l|l|}
\hline Category & Quantity & Proportion \\
\hline cutworms & 1213 & $9.72 \%$ \\
\hline aphidoidea & 1220 & $9.78 \%$ \\
\hline armyw & 1202 & $9.64 \%$ \\
\hline chafer & 1205 & $9.66 \%$ \\
\hline locust & 1225 & $9.82 \%$ \\
\hline $\begin{array}{l}\text { mamestra brassicae } \\
\text { linnaeus }\end{array}$ & 1297 & $10.40 \%$ \\
\hline $\begin{array}{l}\text { psilogramma } \\
\text { menephron }\end{array}$ & 1315 & $10.54 \%$ \\
\hline ostrinia furnacalis & 1207 & $9.68 \%$ \\
\hline clanis bilineata & 1285 & $10.30 \%$ \\
\hline helicoverpa armigera & 1305 & $10.46 \%$ \\
\hline
\end{tabular}

\subsection{Object detection model design}

\subsubsection{Target detection algorithm Faster R-CNN}

Before the appearance of Faster R-CNN, R-CNN and Fast R-CNN already existed, but R-CNN cannot achieve End-to-End training, and Selective Search is more time-consuming. In order to solve these problems, Shaoqing Ren proposed Faster R-CNN ${ }^{[8]}$ target detection algorithm in 2016 NIP. The overall structure of Faster R-CNN is shown in Figure 2. Faster R-CNN integrates feature extraction, proposal extraction, Bounding Box Regression, and Classification into one network, so the speed of target detection has been greatly improved.

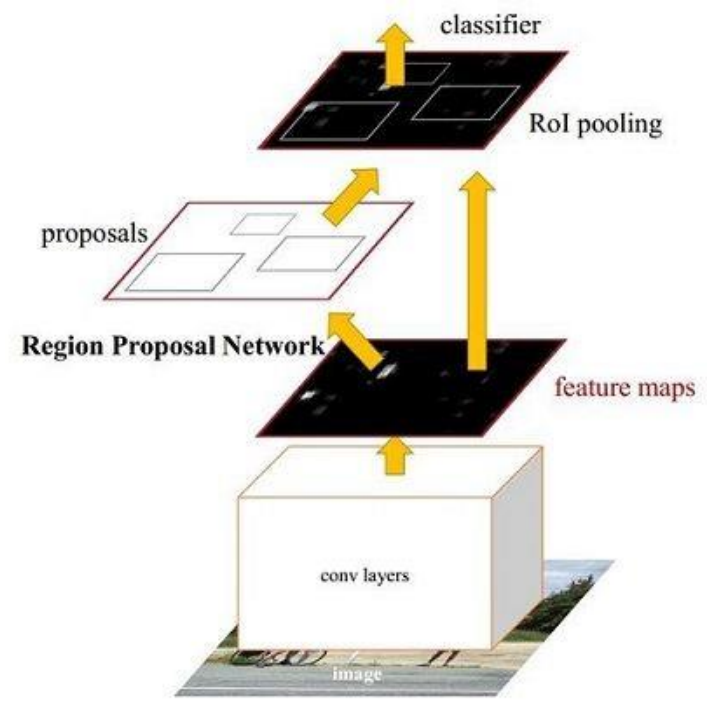

Figure 2. Faster R-CNN overall structure diagram

Regional suggestion network (RPN) ${ }^{[9]}$ is a set that takes an image of any size as input and outputs a rectangular target recommendation box, as shown in Figure 3. 


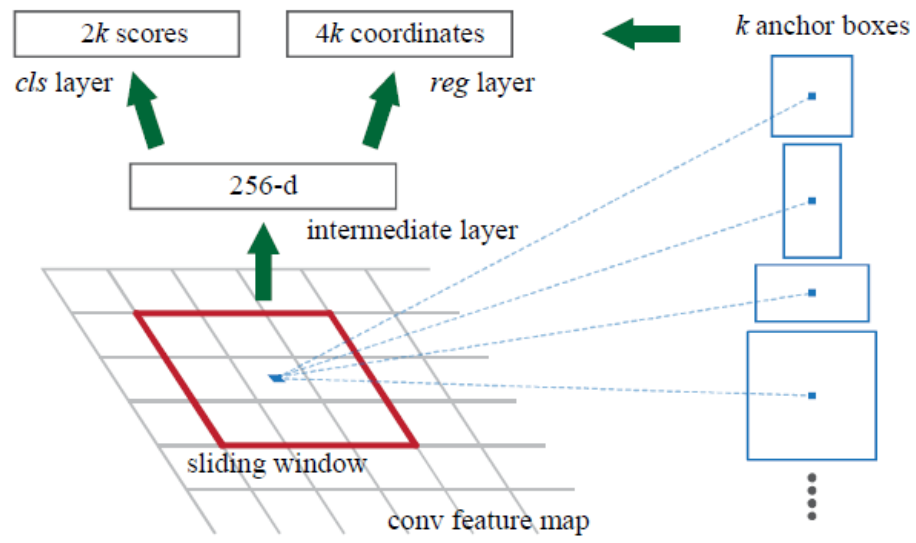

Region Proposal Network (RPN)

Figure 3. Faster R-CNN framework

The regional recommendation network is connected to the last layer of the feature convolution layer. A small $3 \times 3$ network sliding window is used on the feature map of this layer. Only one sliding traversal can extract candidate windows for the entire image, reducing the network calculation burden. The point in the center of the sliding window is called the anchor, and the position of each anchor point can be mapped to the original image, corresponding to the target suggested area on the original image. In order to make the recommendation window meet the target needs without size, the network adopts a multi-scale method, so each sliding window has three scale ratios, and the aspect ratio is 1: 1, 1:2, 2: 1, and 9 types of suggestion windows are generated with three scales, and there are K suggestion windows on the right side of the figure.

As shown on the left side of Figure 3, each sliding window generates a recommendation window, and the recommendation window is mapped to a low-dimensional vector, which is output to two fully connected layers at the same level, bounding box regression layer (reg ) and bounding box classification layer (els). The bounding box regression layer contains the position information of each window. A single suggestion window has four coordinate values to determine the accuracy of the suggestion window generated by RPN. The purpose of the bounding box classification layer is to output the score of the target category of the recommendation window. Each recommendation window has two outputs, corresponding to the probability that the target recommendation window is the foreground / background. For the bounding box generated by the region suggestion network, the part outside the target suggestion region will be discarded, and the remaining regions will be assigned multiple binary labels (target or background). If the highest overlap ratio of the prediction area overlapping the ground truth box (Intersection-overUnion, IoU) is greater than the defined threshold, a positive label is assigned to it. If the IOU ratio of the prediction area is lower than the defined threshold, a negative label is assigned to it. The definition of IoU is as follows:

$$
I o U=\frac{\operatorname{area}\left(B_{\text {in } \sec t} \cap B_{\text {group }}\right)}{\operatorname{area}\left(B_{\text {in } \sec t} \cup B_{\text {group }}\right)}
$$


area $\left(B_{\text {in } \sec t} \cap B_{\text {group }}\right)$ represents the overlapping area of the target recommendation area and the ground truth area, $\operatorname{area}\left(B_{\text {in } \sec t} \cup B_{\text {group }}\right)$ represents the union of the target recommendation area and the ground truth area.

As for the selection of loss function, faster $r$-cnn is the same as fast r-cnn, it follows r.girsick's multi task loss rule ${ }^{[10]}$, According to the definition of bounding box classification layer and bounding box regression layer in RPN, the target classification loss function and the target regression loss function are combined.

$$
L\left(\left\{p_{i}\right\},\left\{p_{i}\right\}\right)=\frac{1}{N_{c l s}} \sum_{i} L_{c l s}\left(p_{i}, p_{i}^{*}\right)+\lambda \frac{1}{N_{\text {reg }}} \sum_{i} p_{i}^{*} L_{r e g}\left(t_{i}, \boldsymbol{t}_{i}^{*}\right)
$$

$N$ is the total amount of anchors, $i$ is the index of anchors in a mini-batch, and $p_{i}$ is the predicted probability of the i-th anchor. If anchor is positive, the ground truth label $p_{i} *$ is 1 , and if anchor is negative, $p_{i} *$ is 0 .

$t_{i}$ represents the four parameterized vectors of the coordinates of the predicted rectangular frame, and $t_{i}^{*}$ is the coordinate vector of the ground truth corresponding to the positive anchor. Classification loss $\left(L_{c l s}\right)$ is the log loss of two categories (foreground and background).

$$
L_{c l s}\left(p_{i}, p_{i}^{*}\right)=-\log \left[p_{i}^{*} p_{i}+\left(1-p_{i}^{*}\right)\left(1-p_{i}\right)\right]
$$

Return loss is rreg $_{\text {: }}$

$$
\operatorname{Lreg}\left(t_{i}, t_{i}^{*}\right)=R\left(t_{i}-t_{i}^{*}\right)
$$

\subsubsection{The problem of gradient disappearance}

Gradient disappearance is a common problem in neural networks, Its performance is to make it difficult for the entire network to adjust the parameters of the previous layers in back propagation. When the network has more layers, the gradient disappears more obviously. This problem is not caused by neural networks, but because the specific activation function adopts a gradient descent method.

For a very simple deep neural network, each layer has only a single neuron. As shown in Figure 4.

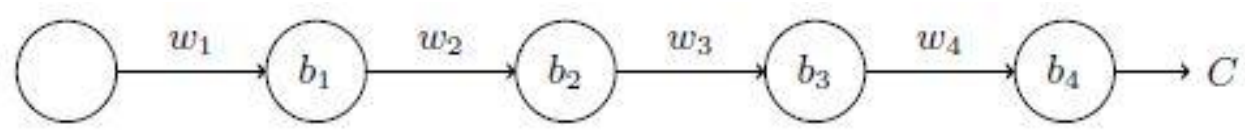

Figure 4. Simple deep neural network

Among them, $\omega_{1,} \omega_{2}$ is the weight, $b_{1}, b_{2}$ is the bias, and $C$ is the cost function.

The result of the cost function $C$ on the partial derivative of the bias $b_{l}$ is calculated as follows: 


$$
\frac{\partial C}{\partial b_{1}}=\sigma^{\prime}\left(z_{1}\right) \times \omega_{2} \times \sigma^{\prime}\left(z_{2}\right) \times \omega_{3} \times \sigma^{\prime}\left(z_{3}\right) \times \omega_{4} \times \sigma^{\prime}\left(z_{4}\right) \times \frac{\partial C}{\partial a_{4}}
$$

Among them, the output of the $j$ th neuron is $a_{j}=\sigma\left(z_{j}\right), z_{j}=\omega_{j} * a_{j-1}+b_{j}$ is the weighted input of the neuron.

When the cost is the sigmoid function, $\sigma^{\prime}(0)=1 / 4$ reaches the highest. If we use the standard method to initialize the weights in the network, we will use a Gaussian distribution with a mean value of 0 and a standard deviation of 1 . So all the weights will usually satisfy $\left|\omega_{j}\right|<1$, so $\omega_{j} \sigma^{\prime}\left(z_{j}\right)<1 / 4$, This creates the problem of gradient disappearance.

In ResNet ${ }^{[10]}$, a residual structure is proposed. The residual structure is shown in Figure 5:

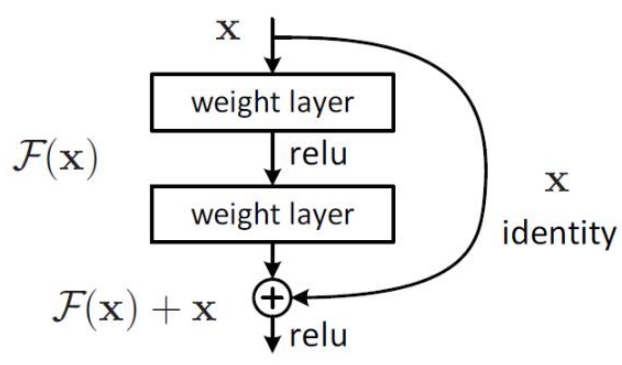

Figure 5. residual structure

This residual module is realized through the fast channel, the input and output of this module are superimposed by a neuron. This superposition has no effect on the amount of network calculations and parameters, but it improves the model's ability. This residual structure can transfer the gradient back to the shallow layer through the shortcut without loss, which can alleviate the gradient disappearance problem.

\subsubsection{Inception network}

This article considers the use of Googlenet Inception ${ }^{[11]}$ structure to build an Inception network. A simple Inception network contains 22 layers of deep network. Because of the problem of blocked information flow, the optimization ability of the deep model is greatly reduced. To solve this problem, Googlenet ${ }^{[12]}$ added two additional Softmax layers to calculate the new loss value, and then recalculated the network gradient based on the new loss value. Ballester ${ }^{[13]}$ proposed the use of Shortcut Connection to reduce the effect of gradient disappearance. This structure can also reduce the Degradation phenomenon. However, some farmland pests have smaller targets. Using the simple concept structure cannot solve the problem of pest detection and recognition in specific situations. Therefore, this paper uses an improved Inception network ${ }^{[14]}$ for farmland pest detection,the improved neural network is shown in Figure 6. 


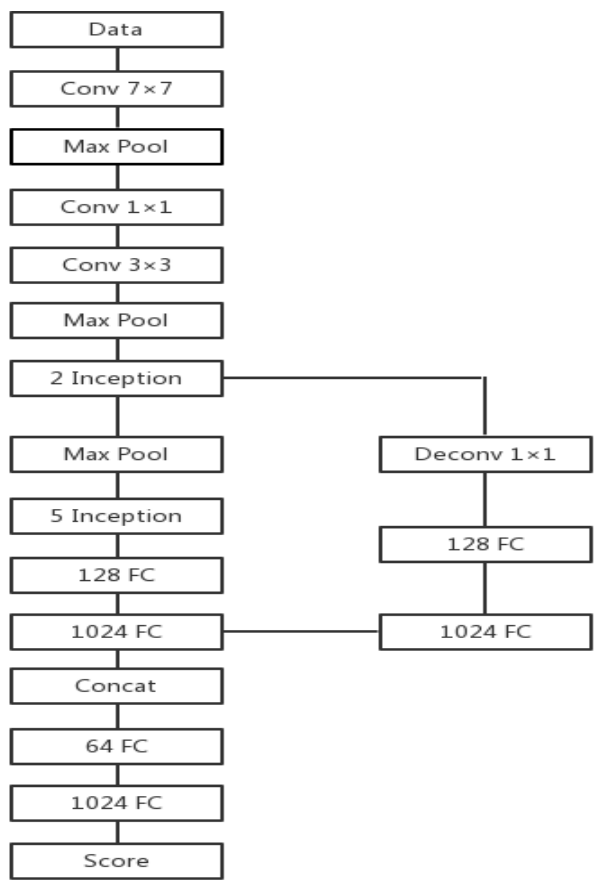

Figure 6. Inception network structure diagram

As can be seen from Figure 6, the improved Inception ${ }^{[14]}$ network uses the Shortcut Connection, and a deconvolution layer is connected behind the second Inception structure, and the feature map is doubled to the original one. At the same time, after extracting the feature map of the seventh perception structure, connecting a full connection layer will reduces the dimension of the feature map to the feature vector of 1024 dimension, Then the two feature vectors are stitched into a 2048-dimensional feature vector, and then the 2048-dimensional feature vector is reduced to a 1024-dimensional feature output as a feature output of the picture. And the Inception network can directly transfer the gradient from the deep layer of the network back to the shallow layer. At the same time, the Shortcut Connection can extract the shallow feature map. The scale of the shallow feature map is $1 / 8$ of the original image. After the deconvolution layer, the feature map is increased to $1 / 4$ of the original image. This kind of network combining multiple layers of features and different scales has better ability to detect and recognize targets, and can get better results in farmland pest recognition.

\subsection{Experimental results and analysis}

\subsubsection{Experimental results}

When using the farm pest database detection model to train, in order to ensure that all samples can be used for training and testing, this paper uses $\mathrm{K}$-fold cross-validation ${ }^{[15]}$, where $\mathrm{K}$ is selected 10 and 9 subsets are selected for training Data, 1 subset as test data.

In this experiment, the network training uses the "pre-training + fine-tuning" method, and uses the pre-trained Inception network model on ImageNet for training fine-tuning. The training adopts conventional hyperparameter configuration: set the weight decay parameter (weight_decay) to 0.0005 , the momentum coefficient (momentum) to 0.9 , the parameter gamma to 0.1 , and the initial learning rate (base_lr) to 0.1 . 
In this experiment, the Xavier method ${ }^{[16]}$ is used to initialize the weight parameters, and the stochastic gradient descent algorithm ${ }^{[17]}$ (SGD) is used for network training.

The results of this experiment were evaluated using mean Average Precision (mAP). The formula is as follows:

$$
m A P=\frac{\sum_{q=1}^{Q} A P(q)}{Q} \times 100 \%
$$

$Q$ is the total number of pest categories, and $A P(q)$ is the average accuracy rate of the detection results of category.

The average accuracy of the experimental results and the final average of the average accuracy are shown in Figure 7.

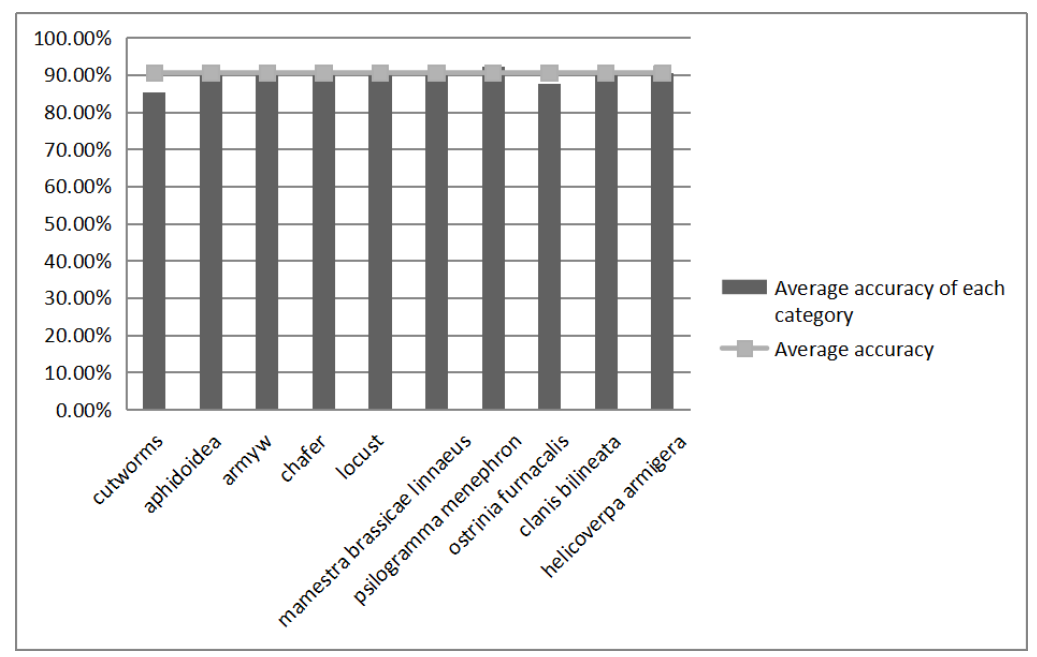

Figure 7. Farm pest detection results

From Figure 7, it can be seen that the average accuracy of detection of most types of farm pests is high, and the average average accuracy can be seen from the broken lines in the figure mAP reached $90.54 \%$.

\subsubsection{Experimental comparison}

In order to evaluate the detection ability of the Faster R-CNN+ Improved Inception network model proposed in this paper, We chose two detection methods for comparison, namely Faster RCNN + VGG16 model and Faster R-CNN + Original Inception model.

In this experiment, the network training also uses the "pre-training + fine-tuning" method, and we adopt the same hyperparameter configuration, initialization algorithm and training algorithm as in Section 2.3.1.

The classification and recognition results of farmland pest images by various network models are shown in Table 2: 
Table 2. Comparison of test results

\begin{tabular}{|l|l|l|l|}
\hline Network model & mAP & $\begin{array}{l}\text { Single image test } \\
\text { duration }\end{array}$ & $\begin{array}{l}\text { Model } \\
\text { size }\end{array}$ \\
\hline Faster R-CNN+VGG16 & $\begin{array}{l}87.85 \\
\%\end{array}$ & $0.030 \mathrm{~s}$ & $512 \mathrm{M}$ \\
\hline $\begin{array}{l}\text { Faster R-CNN+ Original } \\
\text { Inception }\end{array}$ & $\begin{array}{l}85.63 \\
\%\end{array}$ & $0.025 \mathrm{~s}$ & $160 \mathrm{M}$ \\
\hline $\begin{array}{l}\text { Faster R-CNN+ Improved } \\
\text { Inception }\end{array}$ & $\begin{array}{l}90.54 \\
\%\end{array}$ & $0.027 \mathrm{~s}$ & $261 \mathrm{M}$ \\
\hline
\end{tabular}

It can be found that the classification accuracy of the network model used in this paper is improved by about 3\% compared with the classic VGG16 model, and it is improved by 5\% compared to the original Inception network structure. the results show that the model in this paper has achieved good detection results in the farmland pest data set, and the overall classification accuracy has been improved.

\section{Conclusions}

This paper proposes a farmland pest detection model based on target detection algorithm, this model is improved on the basis of perception network architecture, and combines with Faster RCNN target detection algorithm, and test this model with a established farmland pest database. The detection accuracy rate of $90.54 \%$ was obtained. The experimental results show that the recognition model proposed in this paper can well complete the task of farmland pest detection, and improve the detection accuracy and speed. However, the method of this paper still has some shortcomings. The improved Inception network also needs to design a lot of hyper parameters. In the experiments, it is impossible to avoid the complicated hyper parameter tuning process, which brings the risk of over fitting to the recognition model.

\section{ACKNOWLEDGEMENTS}

The successful completion of this article is inseparable from the meticulous guidance and supervision of my tutor, as well as the help and encouragement of the lab brothers and sisters. Thank everyone.

\section{REFERENCES}

[1] Li Y, Xia C , Lee J . Detection of small-sized insect pest in greenhouses based on multifractal analysis[J]. Optik - International Journal for Light and Electron Optics, 2015, 126(19):2138-2143

[2] Geng Ying. Research on crop disease diagnosis based on image recognition[D]. University of Science and Technology of China, 2009.

[3] Li Wenbin. Research on rice pest image recognition technology based on SVM [D]. Hangzhou University of Electronic Science and technology, 2015.

[4] Pan Chunhua, Xiao Deqin, Lin Tanyu, et al. Classification and identification of major vegetable pests in South China based on SVM and regional growth algorithm [J]. Journal of agricultural engineering, 2018 (8): 192-199

[5] Yang Guoguo. Identification and detection of early locust pupae of Chinese rice locust based on machine vision [D]. Zhejiang University, 2017. 
International Journal of Artificial Intelligence and Applications (IJAIA), Vol.11, No.3, May 2020

[6] Yang Wenhan. Research on cotton pest identification system based on digital image processing [D]. Sichuan Agricultural University, 2015.

[7] Qin Fang. Insect image recognition based on deep learning [D]. Southwest Jiaotong University,2018:31-34.

[8] He K, Zhang X, Ren S, et al. Deep Residual Learning for Image Recognition[C]// 2016 IEEE Conference on Computer Vision and Pattern Recognition (CVPR). IEEE Computer Society, 2016.

[9] Xia Denan. Research on agricultural insect image recognition based on deep learning [D]. Anhui University, 2019:26-30.

[10] Girshick R. Fast R-CNN[C]// 2015 IEEE International Conference on Computer Vision (ICCV). IEEE, 2016.

[11] Szegedy C , Liu W, Jia Y, et al. Going Deeper with Convolutions[J]. 2014.

[12] He K , Zhang X, Ren S, et al. Deep Residual Learning for Image Recognition[C]// 2016 IEEE Conference on Computer Vision and Pattern Recognition (CVPR). IEEE Computer Society, 2016.

[13] Ballester P L, Araujo R M . On the performance of GoogLeNet and AlexNet applied to sketches[C]// AAAI. AAAI Press, 2016.

[14] Shen Yufeng.Research on Stored Grain Pest Detection Algorithm Based on Deep Learning[D].2018:36-45.

[15] Hu Juxin,Zhang Gongjie.Selective ensemble classification algorithm based on K-fold cross validation[J].Science and Technology Bulletin,2013(12):123-125.

[16] Shen H. Towards a Mathematical Understanding of the Difficulty in Learning with Feedforward Neural Networks[J]. 2016.

[17] Sutskever I, Martens J, Dahl G, et al. On the importance of initialization and momentum in deep learning[C]// International Conference on International Conference on Machine Learning. JMLR.org, 2013: III-1139.

\section{Authors}

Shi Wenxiu, 1995.03.01, School of Information Science and Engineering, University of Jinan, Postgraduate, Mainly engaged in image processing research. 